\title{
MIIASE HUMANA POR ALOUATTAMYIA BAERI (SHANNON \& GREENE) (DIPTERA, CUTEREBRIDAE). COMUNICAÇÃO DE DOIS CASOS NA REGIÃO NORTE DO BRASIL
}

\section{ABSTRACT}

J.H. GUIMARĀES ${ }^{1}$ CARLOS E.A. COIMBRA JR. ${ }^{2}$

The purpose of this paper is to record two peculiar cases of human myiases occurring in Northern Brasil which apparently have not been reported. In both cases maggots of Alouattamyia baeri (Shannon \& Greene) (Diptera, Cuterebridae) were localized in the pharyngeal cavity. The first case involved a female Surui indian, 30 years old, living in the Parque Aripuanã, Rondônia, presenting symptoms of cough, nausea, dyspnea and painful throat. After treatment with Tiabendazol six large maggots were expelled through the mouth. The second case occurred in the state of Amazonas, involving a 45 years old worker of the Manaus-Manacapuru road, $\mathrm{km}$ 27. The patient showed, for two weeks, intense cough and throat irritation and after gargle with iodine solution expelled a large maggot.

The native hosts of this fly include mainly howler monkeys (Alouatta sp.). The large maggots $(22-27 \mathrm{~mm})$ develop in the cervical, upper sternal and axillary regions. An up-to-date list of hosts of A. baeri found in the literature is presented. The material is deposited in the collection of the Museu de Zoologia, Universidade de São Paulo.

O Prof. Hugo de Souza Lopes, Academia Brasileira de Ciências, recentemente nos enviou para estudo uma interessante larva de Cuterebridae coletada pela equipe de saúde da FUNAI. Tal exemplar, encaminhado ao Prof. Souza Lopes por um dos autores (Carlos E. A. Coimbra Jr.), tinha sido expelido pela boca de uma jovem índia em Rondônia. Logo após, recebemos uma outra amostra de larva de Cuterebridae, proveniente de Manaus, coletada em idênticas condições. Segue-se um resumo dos casos acima.

Caso 1. India Suruí, 30 anos de idade, vivendo no Parque Aripuanã, Rondônia. Atendida pela equipe de saúde da FUNAI, com queixa de que havia "um bicho" subindo pela sua garganta. Ninguém deu muita atenção e ela foi medicada com xaropes, uma vez que estava tossindo.

Nas ocasiões em que ela dizia estar sentindo os "bichos", ficava com dificuldades para respirar e ânsias de vômito. Foi então removida da aldeia e encaminhada à sede do Parque Aripuanã onde ficou sob cuidados médicos na enfermaria. Foi quando expeliu a segunda larva pela boca. Os médicos desconheciam a larva e encaminharam-na para um dos autores (C. E. A. Coimbra Jr.) para identificação. $\mathrm{Na}$ ocasião medicaram-na com Tiabendazol, pois supunham tratar-se de parasitismo intestinal.

Caso 2. J. G. S., 45 anos de idade, sexo masculino. Trabalhador rural no $\mathrm{km} 27$ da rodovia Manaus-Manacaparu. O paciente queixou-se de forte irritação na garganta, tosse intensa durante 10 a 15 dias. Após gargarejo com solução de iodo, expeliu num forte acesso de tosse uma larva de $1,5 \times 0,80 \mathrm{~cm}$ de comprimento. A larva foi encaminhada pelo Sr. Edson Nunes Palheta, Auxiliar Técnico, INPA, Departamento de Ecologia, para identificação.

Comparando as duas amostras com a coleção de larvas do Museu de Zoologia, constatamos de que se tratavam de Alouattamyia baeri (Shannon \& Greene) (Diptera, Cuterebridae). Tal díptero tinha sido registrado na literatura como parasita de certos Primatas, especialmente macacos guaribas (Alouatta sp.).

1. Museu de Zoologia USP. Bolsista do Conselho Nacional de Desenvolvimento Científico e Tecnológico - CNPq.

2. Centro de Estudos e Pesquisa em Antropologia Médica, CEPAM, Brasília, DF. 
As larvas de Cuterebridae se criam no tecido subcutâneo de uma grande variedade de mamíferos, a maioria Rodentia e Lagomorpha, desenvolvendo-se individualmente em cistos abertos ou furúnculos na pele. Quando desenvolvidas abandonam o hospedeiro e caem no solo para pupar. Guimarães (1971) apresentou uma lista dos casos de Cuterebrini citados na literatura, com registros de alguns casos novos.

Alouattamyia baeri ocorre apenas na região neotropical; uma lista atualizada dos Primatas hospedeiros deste díptero aparece na tabela 1. Nestes hospedeiros, larvas grandes $(22-27 \mathrm{~mm})$ desenvolvem-se subcutaneamente na região axilar, cervical e esternal. Os adultos são moscas negras, robustas, com aparência de mamangava. Pouco se sabe da biologia e ecologia deste grupo; Shannon \& Greene (1926) admitiam que os macacos infestavam-se ao ingerir ovos depositados em folhas que lhes serviam de alimentos. As larvas ao serem ingeridas se fixam na faringe do hospedeiro por algum tempo. Após completar certo desenvolvimento, elas penetram mais profundamente nos tecidos do hospedeiro até atingir a região subcutânea, onde formam cistos. Quando atingem a maturidade, as larvas perfuram o cisto caindo no solo para pupar. Goldman (1920) em seus estudos dos mamíferos do Panamá observou: "All the specimens of (Alouatta) obtained carried numerous large larvae of flies, mainly in the skin on the throat which added materially to their repugnant appearance".

Não existe nenhum registro desta espécie parasitando o homem. Na América tropical o único Cuterebridae que ataca regularmente seres humanos causando miíases furunculosas é o "berne" ou "ura" (Dermatobia hominis). Greene (1956: 21) descreveu com detalhes as características das larvas causadoras de miíases nos homens e animais. Segundo este autor, podemos diferenciar a miíase por Dermatobia das de Alouattamyia pelas seguintes características:

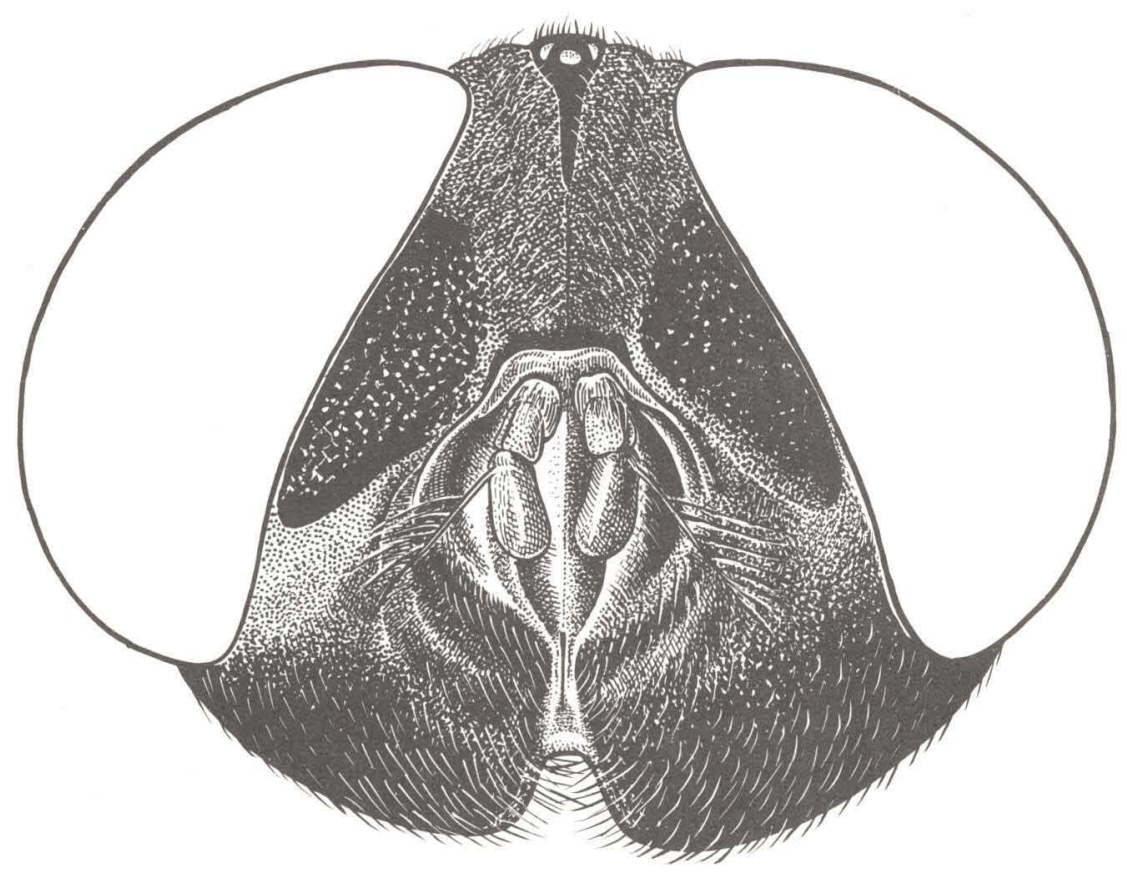

Fig. 1 - Alouatamyia baeri (Shannon \& Greene). Cabeça do macho, vista anterior. Material de Santarém, Pará, Brasil. 
Dermatobia. Larva madura de formato piriforme, coloração testácea, segmentação indistinta. Os segmentos 6 e 7 apresentam fileiras de espinhos fortes, mais numerosas na região dorsal. Tais espinhos apresentam uma base arredondada.

Alouattamyia. Larva de contorno mais ou menos oval, de cor negra, apresentando segmentação bem distinta e o corpo coberto de escamas (fig. 2).

Segundo Greene (1956) as escamas da larva de Alouattamyia apresentam uma fileira irregular de dentes finos em sua superfície livre. Após o exame de larvas de várias procedências, observamos que além das escamas descritas por Greene existem também escamas de bordos lisos, semelhantes a escamas de peixes. Tais escamas são mais numerosas na região dorsal e mediana da larva. O desenho da fig. 2 foi baseado em exemplares de Alouattamyia coletados em guaribas no Pará. As larvas desta amostra medem 2,7 cm x $1,5 \mathrm{~cm}$. As amostras coletadas em seres humanos não atingiram tal desenvolvimento. A larva do caso 1 mede $2 \times 1 \mathrm{~cm}$ e a do caso $2,1,5 \times 0,80 \mathrm{~cm}$.

Acreditamos que o tratamento ocasional desta míase pelo Tiabendazol prescrito pela equipe de saúde do Parque Aripuanã foi bastante feliz e deve ser recomendado em novos casos para a expulsão das larvas fixadas na faringe do paciente. Tal produto é um antihelmíntico de largo espectro, tendo também ação nos estágios imaturos de Oestrus ovis em ovelhas. A solução de iodo usada no caso 2 é contraindicada, principalmente pela ação necrosante do iodo na mucosa oral e da pouca ação inseticida desta substância.

Apresentamos a seguir uma lista atualizada dos Primatas hospedeiros de Alouattamyia baeri registradas na literatura.

TABELA 1. HOSPEDEIROS DE ALOUATTAMYIA BAERI (SHANNON \& GREENE) PRIMATAS

Aotus trivirgatus (Humboldt) (Cebidae, Aotinae) - Guimarães, 1971 (Brasil: Pará).

Alouatta belzebul (Linnaeus) (Cebidae, Alouattinae) - Guimarães, 1971 (Brasil: Maranhão).

Alouatta palliata aequatorialis Festa (como palliata inconsonans Goldman) (Cebidae, Alouattinae) - Ewing, 1925 (Panamá); Zeledón et al., 1957 (Costa Rica).

Alouatta palliata palliata (Gray) (Cebidae, Alouattinae) - Zeledón et al., 1957 (Costa Rica).

Alouatta seniculus (Linnaeus) (Cebidae, Alouattinae) - Shannon \& Greene, 1926 (Guyana e Panamá); Dunn, 1934 (Panamá). O nome correto do macaco, seniculus, foi dado por Hershkovitz (1977: 394).

Alouatta villosa (Gray) (Cebidae, Alouattinae) - Hershkovitz, 1977: 394.

Alouatta sp. (Cebidae, Alouattinae) - Zeledón et al., 1957 (Costa Rica); Shannon \& Greene, 1956 (Guyana); original (Brasil: Pará).

\section{AGRADECIMENTOS}

Os autores ficam gratos aos Drs. Hugo de Souza Lopes, Academia Brasileira de Ciências, ao Sr. Edson Nunes Palheta, Departamento de Ecologia, INPA, Amazonas, pelo encaminhamento do material.

\section{REFERENCIAS}

Dunn, L. H., 1934. Entomological investigations in the Chiriqui region of Panama. Psyche, Cambr. 20: 219-226.

Ewing, N. E., 1925. Cuterebra larvae in skin of Panama howler monkey (Alouatta palliata inconsonans). I. Parasit. 12(2): 107.

Goldmann, E. A., 1920. Mammals of Panama. Smiths. Misc. Coll 69(5): 229.

Greene, C. T., 1956. Dipterous larvae parasite on animals and man and some dipterous larvae causing myiasis in man. Trans. Am. ent. Soc. 82: 17-34. 


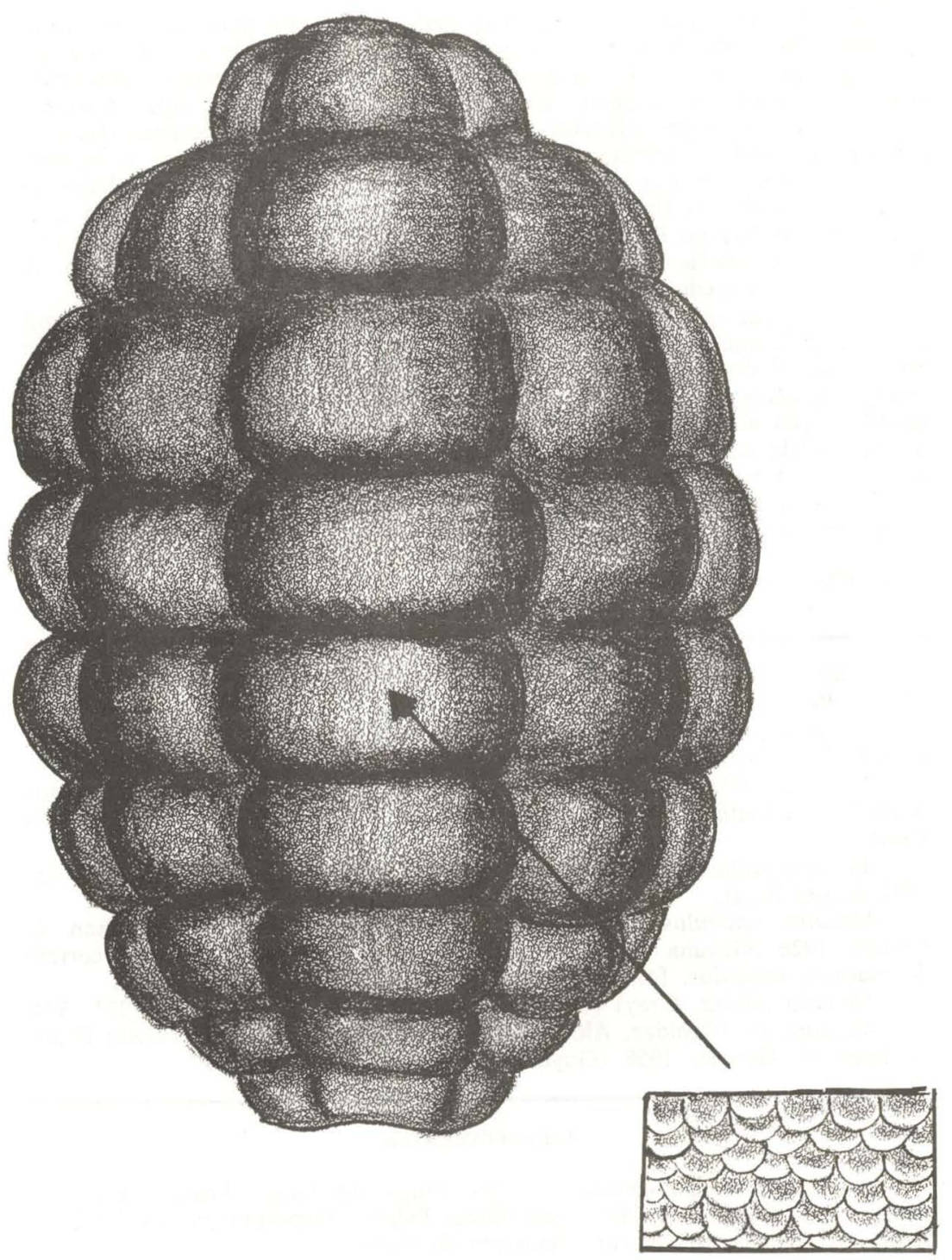

Fig. 2 - Alouatamyia baeri (Shannon \& Greene). Larva III, vista dorsal, com detalhes das escamas da área mediana. Material de Aldeia Araçu, Pará, aumentado $6 \mathrm{X}$. 
Guimarães, J. H., 1971. Note on the hosts of Neotropical Cuterebrini (Diptera, Cuterebridae) with new records from Brazil. Papéis Avulsos Zool., S Paulo 25(10): 84-94.

Hershkovitz, P., 1977. Living New World monkeys (Platyrrhini), with an introduction to Primates, 1: $1117 \mathrm{pp}$. University of Chicago Press.

Shannon, R. C. \& C. T. Greene, 1926. A bot-fly parasitic in monkeys. Zoopathologica, N. York 1: 285-290, 2 figs.

Zeledón, R., O. Jiménez Q. \& R. R. Brenes M., 1975. Cuterebra baeri Shannon \& Greene en el mono aullador de Costa Rica. Revta Biol. trop. 5: 129-134, 11 figs. 